\title{
Efeito da prática de instrumentos musicais nas disfunções temporomandibulares e distúrbios do sono
}

\author{
- Lígia Gabrielle Sanches Mariotto Faculdade de Odontologia, Universidade de São Paulo, Ribeirão Preto, SP, Brasil • Kátia \\ Alessandra de Souza Caetano Programa de Pós-Graduação em Psicologia, Faculdade de Filosofia, Ciências e Letras, \\ Universidade de São Paulo, Ribeirão Preto, SP, Brasil • Carla Larissa Vidal Faculdade de Odontologia, Universidade de São \\ Paulo, Ribeirão Preto, SP, Brasil • Andréa Cândido dos Reis Departamento de Materiais Dentários e Prótese, Faculdade de \\ Odontologia, Universidade de São Paulo, Ribeirão Preto, SP, Brasil
}

RESUMO | Objetivo: Considerando os inúmeros benefícios a saúde física e mental proporcionados pelas atividades artísticas, o objetivo deste estudo é avaliar a relação entre a prática recreativa de instrumentos musicais e as disfunções temporomandibulares (DTM) e os distúrbios do sono (DS) em uma amostra de músicos integrantes de orquestras do interior de São Paulo que praticam instrumentos musicais como forma de lazer. Método: Trata-se de um estudo transversal constituído por dois grupos. O grupo controle, com indivíduos da comunidade em geral, apresentou idade média de 28,35 anos $( \pm 4,867)$, enquanto o grupo de músicos instrumentistas, 28,37 anos $( \pm 6,712)$. Ambos os grupos foram constituídos por 43 voluntários, sendo 14 mulheres e 29 homens. Os participantes responderam ao questionário anamnésico de Fonseca e ao questionário de Fletcher e Luckett para avaliar sintomas de DTM e distúrbios do sono, respectivamente. Resultados: Foi encontrada uma forte associação entre a presença de DTM e o tempo de prática musical, de modo que os participantes com pouca prática possuem $8,57(\mathrm{p}=0,002)$ vezes mais chances de apresentarem DTM do que os participantes com muita prática. Os grupos não diferiram quanto aos sintomas de DTM e DS e quanto a variáveis demográficas. Conclusão: A prática recreativa de instrumentos musicais em longo prazo pode diminuir as chances de desenvolvimento de disfunção temporomandibular. Este estudo reforça que tocar um instrumento musical por lazer traz benefícios para a saúde física, especificamente como fator de proteção ao desenvolvimento de disfunção temporomandibular.

DESCRITORES | Síndrome da Disfunção da Articulação Temporomandibular; Transtornos da Articulação Temporomandibular; Distúrbios do Sono; Sono; Música.

ABSTRACT | Effect of music instruments practice in temporomandibular joint dysfunction and sleep disorders - Objective: The aim of this study was to evaluate the relation among recreational practice of musical instruments, temporomandibular disorders (TMDs), and sleep disturbances in a sample of musicians that are members of orchestras in a southeast area of Brazil. The participants practice playing instruments as a leisure activity. Method: this was a cross-sectional study with two groups. The comparison group included members from the general population. Both group included 43 adults, being 14 women and 29 men. The mean age of comparison group is 28.35 ( \pm 4.867$)$, and the mean age of musician group is $28.37( \pm 6.712)$. The participants completed self-rated questionnaires to assess TMDs symptoms and sleep disturbance. Results: the groups did not differ regarding the presence of TMDs symptoms, sleep disturbance, and demographic variables. A strong and negative correlation was observed between TMDs and time of playing a musical instrument. The participants with less practice in years in playing a musical instrument have 8.57 more chance of having TMDs than the musicians with a long history of playing an instrument. Conclusion: it seems that a long history of practicing a musical instrument as a leisure activity is associated with a lower chance of developing TMDs. This study reinforces the benefits of playing a musical instrument as a leisure activity to physical health, specifically, as a protective factor to developing TMDs.

DESCRIPTORS | Temporomandibular Joint Dysfunction Syndrome; Temporomandibular Joint Disorders; Sleep Disorders; Sleep; Music.

AUTOR CORRESPONDENTE

- Andréa Cândido dos Reis Departamento de Materiais Dentários e Prótese, Faculdade de Odontologia, Universidade de São Paulo • Av. do Café, s/no $\mathbf{n}^{\circ}$ Monte Alegre Ribeirão Preto, SP, Brasil • 14040-904 E-mail: andreare73@yahoo.com.br

- Recebido 3 ago 2015 - Aceito 19 out 2015

- Dol http://dx.doi.org/10.11606/issn.2357-8041.clrd.2015.122872 


\section{INTRODUÇÃO}

As disfunções temporomandibulares (DTMs) são distúrbios funcionais e estruturais do sistema estomatognático que envolvem os músculos da mastigação e as articulações temporomandibulares (ATMs). ${ }^{1}$ Seus sinais e sintomas podem ser facilmente confundidos com outras desordens ou doenças. No entanto, geralmente acarretam dor na região pré-auricular, limitação ou assimetria de movimentos mandibulares e sons articulares. Além de poderem gerar sintomas secundários auditivos, dores referidas ou cefaleias. ${ }^{2}$

Da população em geral, 40-75\% das pessoas têm algum sinal de DTM e 33\% possuem ao menos um sintoma. ${ }^{3}$ Além disso, DTMs são a segunda causa mais comum de dor orofacial, precedida apenas pela dor de origem dentária. ${ }^{4}$

Além dos sinais e sintomas característicos, a DTM pode estar relacionada a alterações na qualidade do sono. ${ }^{5}$ Os distúrbios do sono (DS) provocam estado de vigília durante a noite, resultando em sensação de sono agitado, sonolência diurna, estresse psicológico e fadiga crônica, além de comprometerem o humor, a atenção, a memória, provocar irritação e acentuar problemas familiares e sociais. ${ }^{6}$ No entanto, apesar de serem tão comuns quanto asma e diabetes, são pouco diagnosticados e tratados adequadamente. ${ }^{7,8}$

A etiologia dos DS é multifatorial e varia desde questões emocionais (depressão, ansiedade), sociais (desemprego, violência), físicas (dor crônica) e ambientais (ruídos internos e externos ao quarto de dormir). ${ }^{9}$ Quando prolongados, os distúrbios do sono podem influenciar profundamente a qualidade de vida e a saúde e aumentar o risco de mortalidade. ${ }^{10,11}$

\section{JUSTIFICATIVA}

Buscando alternativas de tratamento para alterações tão complexas que acabam por interferir na qualidade de vida dos pacientes, formulou-se a hipótese da influência da prática recreativa de instrumentos musicais nos sinais e sintomas de DTM e distúrbios do sono, proporcionando melhora na saúde física e mental.

\section{OBJETIVOS}

O objetivo primário deste estudo foi avaliar a influência da prática recreativa de um instrumento musical nos sinais e sintomas de DTMs e DS por meio do questionário Anamnésico, de Fonseca et al., ${ }^{12}$ e do questionário de Fletcher e Luckett. ${ }^{13}$

Os objetivos secundários foram:

a. Avaliar a prevalência de DTM e DS em músicos instrumentistas e na população em geral

b. Avaliar se há associação entre DS e DTM.

c. Avaliar a influência do tempo de prática musical nos sinais e sintomas de DTM e DS.

\section{MATERIAIS E MÉTODOS}

\section{Local e população da pesquisa}

Este foi um estudo transversal que avaliou a prevalência de desordens temporomandibulares e distúrbios do sono em uma amostra de adultos do interior de São Paulo. A pesquisa incluiu 86 participantes igualmente divididos em dois grupos: um grupo de Músicos Instrumentistas (MI) composto por 43 membros da Orquestra Jazz Sinfônica, situada no município de São João da Boa Vista (SP) e da Orquestra Filarmônica de Santa Rosa de Viterbo (SP); e um grupo de comparação constituído por 43 indivíduos da população de São João da Boa Vista (SP) que não praticam instrumentos musicais. Esses participantes foram pareados por idade, sexo e nível educacional com o grupo de músicos instrumentistas.

Seguindo os critérios de inclusão preestabelecidos, participaram do estudo pessoas na faixa etária de 18 a 40 anos. No caso dos músicos, exigiu-se um mínimo de três anos de experiência.

Não puderam participar do estudo sujeitos que apresentaram alguma deficiência grave de coluna ou postural, deficiência mental ou alguma outra anormalidade.

\section{Instrumento de coleta de dados}

O instrumento de coleta de dados foi composto pelos seguintes questionários (Anexo 1):

a. Questionário de história de hábitos e comportamento, que contempla as variáveis sexo, idade, 
profissão, problemas médicos. No grupo MI, também foram abordadas as variáveis tipo de instrumento, horas semanais dedicadas à atividade, há quanto tempo estuda música, além de problemas de saúde relacionados à prática.

b. Questionário adaptado de Fletcher e Luckett, ${ }^{13}$ que compreende 38 questões relacionadas ao sono e queixas associadas. Cada uma das 38 questões é pontuada de o a 3 (nunca $=0$, muito raramente $=1$, ocasionalmente $=2$, frequentemente $=3$ ); o escore global varia de o a 114, sendo dividido por 38 . Pontuações $>1$ sinalizam a presença de sintomas significativos de distúrbios do sono.

c. Questionário anamnésico de Fonseca et al., ${ }^{12}$ que consiste em dez questões sobre dores na ATM, pescoço ou ouvidos, ruídos ou dificuldade em movimentar a mandíbula e hábitos parafuncionais. Para cada uma das questões do questionário de Fonseca são possíveis três respostas (sim, não e às vezes) para as quais são preestabelecidas três pontuações (10, o e 5, respectivamente). Com a somatória dos pontos atribuídos obtémse um índice anamnésico que permite classificar os voluntários em categorias de acordo com a severidade dos sintomas: sem DTM (o a 15 pontos), DTM leve (20 a 45 pontos), DTM moderada (50 a 65) e DTM severa (70 a 100 pontos).

\section{Aspectos legais e éticos da pesquisa}

O projeto de pesquisa foi apresentado ao Comitê de Ética em Pesquisa da Faculdade de Odontologia de Ribeirão Preto (FORP) e aprovado sob o protocolo de número 50415315.8.0000.5419. Os indivíduos que aceitaram participar da pesquisa assinaram o Termo de Consentimento Livre e Esclarecido e foram informados sobre os objetivos do estudo, a liberdade em participar ou não, além da garantia de sigilo total das informações coletadas.

\section{Análise estatística}

Foi verificada a normalidade dos dados por meio do teste de Shapiro-Wilk. Para os dados com distribuição normal, foi aplicado o teste T, e para os dados sem distribuição normal, foi realizado o teste U de Mann-Whitney. Também foi realizada uma análise pelo teste Qui Quadrado a fim de verificar a associação entre variáveis qualitativas.

\section{RESULTADOS}

Não houve diferença entre os grupos avaliados quanto às variáveis gênero $\left[\mathrm{X}^{2}(1)=0,000, \mathrm{p}>0,05\right]$ e idade $[\mathrm{t}(76,58)=0,018, \mathrm{p}>0,05]$, sendo a idade média do grupo instrumentista de 28,37 anos (DP=6,712) e a do grupo controle de 28,35 anos $(\mathrm{DP}=4,864)$. Com relação ao tipo de instrumento tocado, $51,16 \%$ dos músicos relataram praticar instrumentos de corda, 30,23\% instrumentos de sopro, 6,98\% instrumentos de percussão e 9,30\% praticam instrumentos de sopro e de corda.

Os instrumentistas possuem um tempo médio de prática de instrumento musical de 14,07 anos $(\mathrm{DP}=7,25)$, sendo que a amostra varia entre 5 e 33 anos de prática, com mediana de 13 anos. Posteriormente, os instrumentistas foram divididos em dois subgrupos, conforme o tempo médio de prática musical: o grupo com pouca prática, formado por aqueles indivíduos que apresentaram um tempo de prática musical menor que a média do grupo; e o grupo com muita prática musical, formado por aqueles indivíduos que apresentaram um tempo de prática de instrumento maior que a média do grupo. Em relação à quantidade de horas semanais despendidas à prática do instrumento, os participantes variam de 2 a 40 horas semanais, com tempo médio semanal de 16,58 horas ( $\mathrm{DP}=10,67)$.

Para avaliar se essas duas variáveis (tempo de prática do instrumento musical e quantidade de horas semanais despendidas à prática do instrumento) possuem distribuição normal, foi realizado o teste de normalidade, que observou normalidade dos dados. Assim, a divisão dos instrumentistas em dois grupos conforme o tempo de prática do instrumento musical, utilizando, para isso, a média como ponto de corte, foi mantida nas análises sequentes.

Em relação ao questionário anamnésico de Fonseca, que considera como indicativo de DTM valores iguais ou superiores a 20 pontos, o escore 
médio do grupo instrumentista é de 19,77 (DP=2,12) e do grupo controle é de 20,09 ( $\mathrm{DP}=2,93)$. Não foi encontrada diferença estatisticamente significante entre os grupos ( $U=831,000, p>0,05)$.

Já no que se refere ao questionário de Fletcher e Luckett, ${ }^{13}$ que permite avaliar distúrbios do sono por meio dos escores dos respondentes, também não foi encontrada diferença estatisticamente significante ( $U=837,000, p>0,05)$. O grupo instrumentista possui escore médio de $0,63(\mathrm{DP}=0,36)$ e o grupo controle possui escore médio de 0,58 $(\mathrm{DP}=0,30)$. É válido salientar que escores iguais ou superiores a um são indicativos de presença de distúrbios do sono.

Dentre as cinco categorias distintas em relação ao sono - qualidade do sono ( $U=917,000, p>0,05)$, sonolência diurna ( $\mathrm{U}=784,00, \mathrm{p}>0,05)$, ronco $(\mathrm{U}=823,000, \mathrm{p}>0,05)$ apneia $(\mathrm{U}=876,500, \mathrm{p}>0,05)$ e queixas associadas $[\mathrm{t}(84)=0,989, \mathrm{p}>0,05]-$ nenhuma delas apresentou diferença estatisticamente significante entre os grupos. A Tabela 1 apresenta o escore médio e desvio padrão em cada um dos domínios, juntamente com o escore geral de distúrbio do sono e da ATM.

TABELA 1 Escores médios dos participantes instrumentistas e controles em diferentes questionários e em relação ao número médio de horas de sono e tempo médio em minutos para iniciar o sono e a estatística de comparação entre os grupos conforme os diferentes sintomas

\begin{tabular}{l|c|c|c} 
& Instrumentistas & Controle & Estatística \\
\hline Distúrbios da ATM & $19,77( \pm 2,12)$ & $20,09( \pm 2,93)$ & $U=831,000(p>0,05)$ \\
Distúrbios do sono & $0,63( \pm 0,36)$ & $0,58( \pm 0,30)$ & $U=837,000(p>0,05)$ \\
Sono & $8,53( \pm 5,83)$ & $7,84( \pm 3,88)$ & $U=917,000(p>0,05)$ \\
Sonolência diurna & $4,12( \pm 3,49)$ & $3,16( \pm 2,91)$ & $U=784,000(p>0,05)$ \\
Ronco & $4,67( \pm 3,95)$ & $4,07( \pm 3,85)$ & $U=823,000(p>0,05)$ \\
Apnéia & $0,53( \pm 1,16)$ & $0,35( \pm 0,72)$ & $U=876,500(p>0,05)$ \\
Queixas associadas & $7,77( \pm 5,39)$ & $6,67( \pm 4,83)$ & $t=0,98$ \\
\hline
\end{tabular}

Também não foi observada correlação entre os escores no questionário de distúrbio do sono e no de distúrbio da ATM (rS=-0,159, p>0,05).

Entre os músicos instrumentistas, observou-se que $62,8 \%$ (27 participantes) apresentam distúrbio da ATM. Entre estes, 74,1\% (20 participantes) possuem pouca prática musical e 25,9\% (7 participantes) muita prática musical. De acordo com o teste de qui-quadrado, há uma associação significativa entre apresentar distúrbio da ATM e o tempo de prática musical, sendo observado que os participantes que possuem pouca prática musical apresentam significativamente maior incidência de distúrbio da ATM do que os participantes com muita prática musical $\left[X^{2}(1)=9,811,(p=0,002)\right]$. Baseado no risco relativo, os participantes com pouca prática têm 8,57 mais chances de apresentarem distúrbio da ATM do que os participantes com muita prática. A distribuição de frequências entre estes grupos pode ser observada no Gráfico 1.

\section{DISCUSSÃO}

Este estudo avaliou a influência da prática musical como atividade recreativa nos sinais e sintomas característicos de DTM e DS. A amostra foi composta por músicos instrumentistas de orquestras do interior de São Paulo e indivíduos da comunidade em geral que não praticam atividade musical.

Foi encontrada uma forte associação entre a presença de DTMs e o tempo de prática musical, sendo observado que os participantes com pouca prática têm 8,57 vezes mais chances de apresentarem DTM do que os participantes com muita prática $(p=0,002)$. Em relação à quantidade de horas semanais dedicadas ao instrumento, os grupos não 
GRÁFICO 1 || Distribuição de frequência entre os grupos pouca prática musical e alta prática musical em relação a presença ou ausência de distúrbio da ATM.

\section{UIsturbIo da A I M}

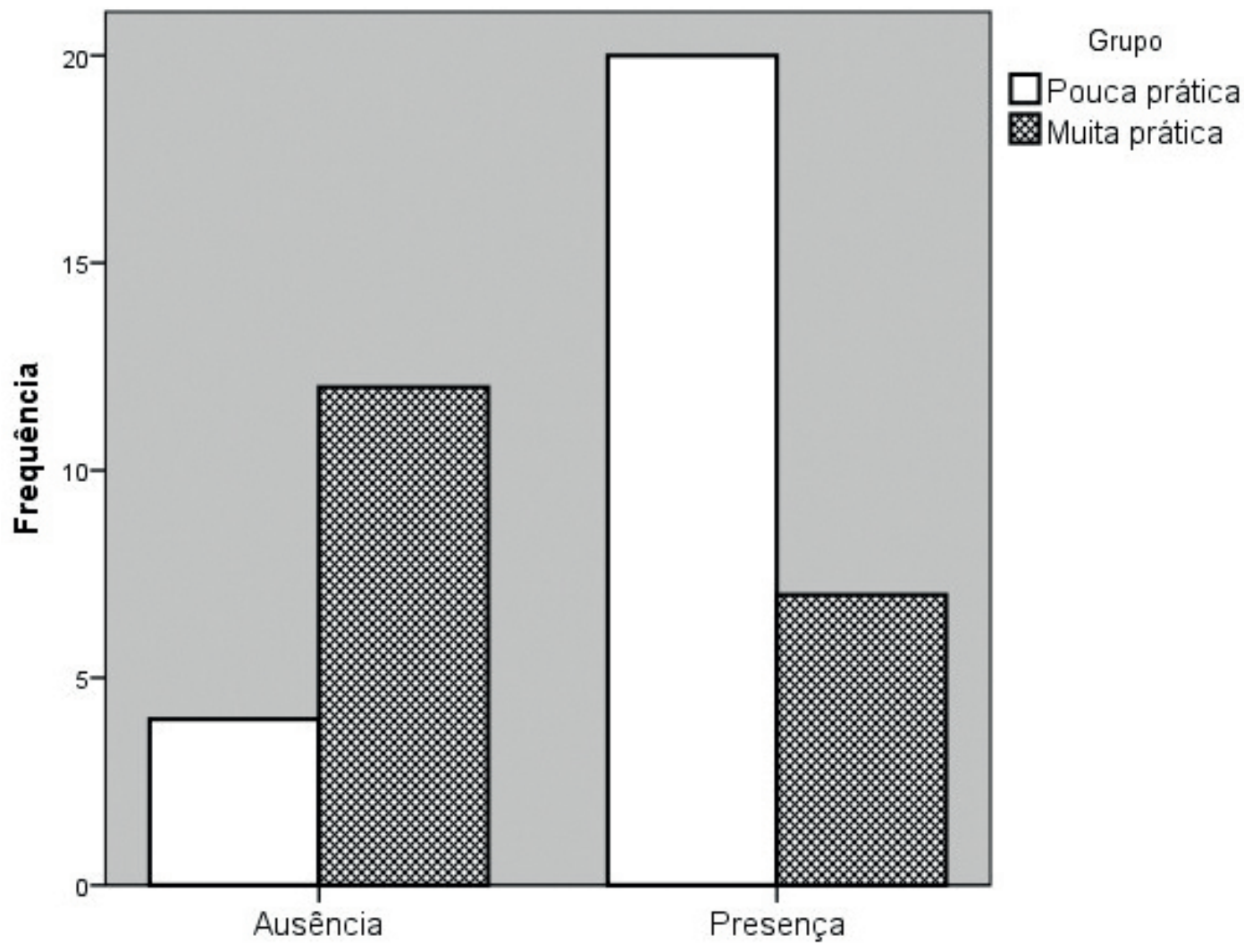

diferem estatisticamente entre si ( $\mathrm{p}>0,05)$, portanto, a frequência semanal não influenciou tal associação. O resultado pode ser explicado pela capacidade da música de controlar o estresse e promover experiências de recompensa, prazer e inserção social..$^{14-16}$

O aumento da tensão muscular decorrente da ansiedade e estresse pode promover o desenvolvimento de DTM, ${ }^{17-19}$ visto que estudos comprovam que indivíduos com níveis elevados de ansiedade e depressão possuem maior risco de desenvolverem DTM em comparação a indivíduos com níveis inferiores desses sintomas. ${ }^{17,20}$ Chisnoiu et al., ${ }^{21}$ também sugere que a ansiedade e o estresse fazem parte da etiologia da DTM e desempenham um papel significativo em manter os sintomas ou piorá-los.

Portanto, como a DTM possui diversas etiologias, entre elas os estressores psicossociais, atividades prazerosas, como a prática recreativa de um instrumento musical (por objetivarem, direta ou indiretamente, a redução dos níveis de ansiedade e estresse), podem ser úteis para diminuir os sinais e sintomas de DTM ou até mesmo impedir seu desenvolvimento. ${ }^{1}$

Embora a prática de um instrumento musical possa ser considerada uma atividade relaxante, há dúvidas quanto à eficácia dessa atividade na diminuição dos distúrbios temporomandibulares, uma vez que há indícios que instrumentistas profissionais estão expostos a riscos ocupacionais que os predispõem a problemas musculoarticulares e a dores, desvios posturais, problemas auditivos, entre outros..$^{22,23}$

A elevada carga horária dedicada ao aperfeiçoamento técnico, posturas incorretas ao tocar, inadequações anatômicas dos instrumentos musicais, além de ensaios e apresentações em 
horários tardios são algumas características do trabalho desses profissionais que podem contribuir para um aumento do risco de desenvolvimento de patologias como distúrbios da ATM e do sono, reduzindo a qualidade de vida e a capacidade laboral. ${ }^{24}$

A amostra foi composta majoritariamente por instrumentistas de corda e sopro. Entre os músicos, violinistas e violistas relatam sentir dores no pescoço mais frequentemente que indivíduos da população em geral, 40\% e 14\%, respectivamente. ${ }^{25}$ Essa classe também é mais propensa a apresentar sinais e sintomas de DTM como dor nos músculos masseter e temporal e especificamente dor na região da ATM direita, devido à pressão sofrida pela mandíbula e músculos mastigatórios ao segurar o instrumento por longos períodos. ${ }^{26} \mathrm{~A}$ DTM também apresenta alta prevalência em instrumentistas de sopro, pois a protrusão da mandíbula necessária para a embocadura do instrumento pode exigir forças excessivas da região da ATM, induzindo a sobrecarga das estruturas a ela relacionadas. ${ }^{25}$

A literatura relata, ainda, que há relação entre DS e DTM em membros de orquestras. ${ }^{27}$ De modo que a baixa qualidade do sono afeta o indivíduo tanto física quanto psicologicamente, causando desde diminuição na capacidade de desempenhar as atividades do dia a dia até alteração na percepção de dor e desconforto. ${ }^{28}$

No entanto, possivelmente devido à amostra ser composta por músicos amadores, não foi encontrada diferença estatisticamente significante entre os grupos avaliados, fato que sugere que a prática de um instrumento musical não interfere negativamente ou promove o aumento dos sinais e sintomas de DS e DTM.

Em suma, a prática regular de um instrumento musical como atividade recreativa pode ter função terapêutica, pois além de promover a saúde mental mediante a melhora nos níveis de ansiedade e estresse, também promove a saúde física ao diminuir as chances de se desenvolver DTM. ${ }^{29}$ Desse modo, estudos futuros devem explorar, quer por meio da utilização de ensaios clínicos randomizados ou estudos longitudinais, os efeitos da prática musical na DTM, manipulando as variáveis entre grupos diferentes.

\section{CONCLUSÃO}

De acordo com a metodologia aplicada, os resultados sugerem que a prática recreativa da atividade musical por um longo período pode diminuir as chances de desenvolvimento de disfunção temporomandibular. No entanto, estudos mais aprofundados podem trazer outras conclusões sobre a intensidade do benefício da pratica musical para a redução de sinais e sintomas de DTM.

\section{REFERÊNCIAS}

1. Conti PC, Corrêa AS, Lauris JR, Stuginski-Barbosa J. Management of painful temporomandibular joint clicking with different intraoral devices and counseling: a controlled study. J Appl Oral Sci. 2015;23(5):529-35. doi: 10.1590/1678775720140438.

2. Porto De Toledo I, Stefani FM, Porporatti AL, Mezzomo LA, Peres MA, Flores-Mir C, et al. Prevalence of otologic signs and symptoms in adult patients with temporomandibular disorders: a systematic review and meta-analysis. Clin Oral Investig. 2017;21(2):597-605. doi: 10.1007/s00784016-1926-9.

3. De Leeuw R. Orofacial pain: guidelines for assessment, diagnosis and management. 4th ed. Chicago: Quintessence; 2008

4. Manfredini D, Guarda-Nardini L, Winocur E, Piccotti F, Ahlberg J, Lobbezoo F. Research diagnostic criteria for temporomandibular disorders: a systematic review of axis I epidemiologic findings. Oral Surg Oral Med Oral Pathol Oral Radiol Endod. 2011;112(4):453-62. doi: 10.1016/j.tripleo.2011.04.021.

5. Rener-Sitar K, John MT, Pusalavidyasagar SS, Bandyopadhyay D, Schiffman EL. Sleep quality in temporomandibular disorder cases. Sleep Med. 2016;25:105-112. doi: 10.1016/j. sleep.2016.06.031.

6. Lima J, Rovere H, Rossini S, Angelis G, Inocente N, Lima A, et al. Prejuízo no sono e recolocação profissional. In: Reimão R. Sono: atualidades. São Paulo: Associação Paulista de Medicina; 2006. p. 222-3. 
7. Benca RM. Diagnosis and treatment of chronic insomnia: a review. Psychiatr Serv. 2005;56(3):332-43. doi: 10.1176/appi. ps.56.3.332.

8. Souza JC, Reimão R. Epidemiologia da insônia. Psicol Estud. 2004;9(1):3-7. doi: 10.1590/S1413-73722004000100002.

9. Rossini S, Reimão R. Insônia: avaliação e prática clínica. In: Reimão R, Rossini S, Valle L. Segredos do sono: sono e qualidade de vida. Ribeirão Preto: Tecmedd; 2008. p. 65-75.

10. Inocente CO, Inocente JJ, Inocente NJ, Reimão R. Estresse, Bournot e sono em professores. In: Reimão R. Medicina do sono, desafios para o século XXI. São Paulo: Associação Paulista de Medicina; 2009.

11. Linton SJ. Does work stress predict insomnia? A prospective study. Br J Health Psychol. 2004;9(Pt 2):127-36. doi: 10.1348/135910704773891005.

12. Fonseca DM, Bonfante G, Valle AL, Freitas SFT. Diagnóstico pela anamnese da disfunção craniomandibular. RGO (Porto Alegre). 1994;42(1):23-4, 27-8.

13. Fletcher EC, Luckett RA. The effect of positive reinforcement on hourly compliance in nasal continuous positive airway pressure users with obstructive sleep apnea. Am Rev Respir Dis. 1991;143(5 Pt 1):936-41.

14. Baadjou VA, Roussel NA, Verbunt JA, Smeets RJ, de Bie RA. Systematic review: risk factors for musculoskeletal disorders in musicians. Occup Med (Lond). Epub 2016 May 2. doi: 10.1093/occmed/kqwo52.

15. Chanda ML, Levitin DJ. The neurochemistry of music. Trends Cogn Sci. 2013;17(4):179-93. doi: 10.1016/j.tics.2013.02.007.

16. Kreutz G. The value of music for public health. In: Clift $S$, Camic PM. Oxford textbook of creative arts, health, and wellbeing: international perspectives on practice, policy and research. Oxford: Oxford University Press; 2015. p. 211-7.

17. Kindler S, Samietz S, Houshmand M, Grabe HJ, Bernhardt O, Biffar R, et al. Depressive and anxiety symptoms as risk factors for temporomandibular joint pain: a prospective cohort study in the general population. J Pain. 2012;13(12):1188-97. doi: 10.1016/j.jpain.2012.09.004.

18. Minghelli B, Kiselova L, Pereira C. Associação entre os sintomas de disfunção temporo-madibular com factores psicológicos e alterações na coluna cervical em alunos da Escola Superior de Saúde Jean Piaget do Algarve. Rev Port Saúde Pública. 2011;29(2):140-7. doi:10.1016/So8709025(11)70018-9.
19. Moraes AR, Sanches ML, Ribeiro EC, Guimarães AS. Therapeutic exercises for the control of temporomandibular disorders. Dental Press J Orthod. 2013;18(5):134-9.

20. Giannakopoulos NN, Keller L, Rammelsberg P, Kronmüller KT, Schmitter M. Anxiety and depression in patients with chronic temporomandibular pain and in controls. J Dent. 2010;38(5):369-76. doi: 10.1016/j.jdent.2010.01.003.

21. Chisnoiu AM, Picos AM, Popa S, Chisnoiu PD, Lascu L, Picos A, et al. Factors involved in the etiology of temporomandibular disorders - a literature review. Clujul Med. 2015;88(4):473-8. doi: 10.15386/cjmed-485.

22. Stechman J Neto, Almeida C, Bradasch ER, Corcoletti LCBJ, Silvério KC, Pontes MMA, et al. Ocorrência de sinais e sintomas de disfunção temporomandibular em músicos. Rev Soc Bras Fonoaudiol. 2009;14(3):362-6. doi: 10.1590/S151680342009000300012.

23. Steinmetz A, Zeh A, Delank KS, Peroz I. Symptoms of craniomandibular dysfunction in professional orchestra musicians. Occup Med (Lond). 2014;64(1):17-22. doi: 10.1093/occmed/ kqt148.

24. Jacukowicz A. Psychosocial work aspects, stress and musculoskeletal pain among musicians: a systematic review in search of correlates and predictors of playing-related pain. Work. 2016;54(3):657-68. doi: 10.3233/wor-162323.

25. Głowacka A, Matthews-Kozanecka M, Kawala M, Kawala B. The impact of the long-term playing of musical instruments on the stomatognathic system: review. Adv Clin Exp Med. 2014;23(1):143-6.

26. Amorim MI, Jorge AI. Association between temporomandibular disorders and music performance anxiety in violinists. Occup Med (Lond). 2016;66(7):558-63. doi: 10.1093/ occmed/kqwo8o.

27. Brown DL, Zahuranec DB, Majersik JJ, Wren PA, Gruis KL, Zupancic M, et al. Risk of sleep apnea in orchestra members. Sleep Med. 2009;10(6):657-6o. doi: 10.1016/j. sleep.2008.05.013.

28. Reimão R, Valle L, Valle E, Rossini S. Sono e saúde: interfaces com neurologia e psicologia. Ribeirão Preto: Novo Conceito; 2010.

29. Ekholm O, Juel K, Bonde LO. Associations between daily musicking and health: results from a nationwide survey in Denmark. Scand J Public Health. Epub 2016 Aug 16. doi: 10.1177/1403494816664252. 